\title{
Reshaping mental health practice with evidence: the Mental Health Research Network
}

\begin{abstract}
Many psychiatrists will have searched for the solution to a clinical problem only to find that the evidence uncovered was grossly inadequate or thoroughly confusing. Some will have felt that such uncertainty is part of the science of psychiatry, but we would argue that it arises from deficits in the quantity and quality of the underpinning research.
\end{abstract}

\section{The scale and quality of psychiatric research}

Even at a superficial level, research in mental health appears to fall behind what might be expected from the disease burden. For example, the World Health Organization estimates that mental disorders account for $12.3 \%$ of the global burden of disease (approximately $20 \%$ in developed countries) (Thornicroft et al, 2002), but less than 5\% (17 558 of 375143) of trials in the Cochrane Controlled Trials Register are indexed under 'mental disorder'. Deeper examination reveals the type and geographical distribution of research. When the Cochrane Schizophrenia Group carried out a survey of 2000 controlled trials relating to schizophrenia research they found the literature dominated by hospital-based drug trials undertaken in the United States (Thornley et al, 1998). Although schizophrenia is not a short-term disorder, over half of the trials ( $N=1082)$ lasted for no more than 6 weeks and only one-fifth lasted longer than 6 months ( $N=382$ ). Only $3 \%$ of trials had sufficient power to detect a clinically important improvement in mental state and over half had fewer than 50 participants, with the trial size not increasing over time.

The small-scale and short-term nature of most psychiatric trials makes it difficult to address questions of clinical relevance. Although service users tend to base their treatment preferences on an evaluation of risks and benefits, few psychiatric trials have sufficient power to evaluate such risks adequately. Hence, the mental health research community was slow to realise the importance of medication side-effects such as sexual impairment and weight gain. Similarly, few psychiatric trials have much to say about the benefits or risks to particular sub-groups (women or ethnic minorities) or sub-populations (urban versus rural). Yet, given the complexity of mental health interventions, it would be astonishing if such differences did not exist. With an evidence base of this quality, it is perhaps not surprising that 75 of 109 recommendations in the NICE Schizophrenia Guidelines (National Institute for Clinical Excellence, 2002) were based on the clinical experience of the Guidelines Development Group or on expert committee reports (the two lowest grades of evidence as defined by the Guidelines Development Group).

The deficit in the quantity and quality of mental health research is related to a deficit in the funding of mental health research. For example, in 1999-2000 out of a total research budget of $f 318.4$ million, the Medical Research Council spent $f 17.6$ million (5.5\%) on mental health research, a proportion considerably less than would be expected from the predicted burden of disease. A similar gap is obvious in the USA. A recent report suggested that only $28.5 \%$ of National Institute of Mental Health (NIMH) awards are in the area of serious mental illness, despite the fact that $58 \%$ of all direct care costs are to this group (Torrey et al, 2003). In our view, this funding deficit does not reflect unfairness in the grant awarding process so much as a quality gap between mental health grant applications and those received from competing disciplines.

Recent Government reports (Office of Science and Technology, 2003) and the Academy of Clinical Medicine (2003) have suggested that one solution to small-scale research, and perhaps to the timeliness of research, is to develop infrastructures or networks to support largerscale studies. The National Institute of Mental Health for England (NIMHE) had the forethought, together with the Department of Health Research and Development, to draw up the blueprint for such a network, which now exists for supporting trials and projects in mental health. The network is called the Mental Health Research Network (MHRN) and this organisation aims to close the quality and the funding gap between mental health and other branches of medical research.

The MHRN is run by a managing partnership of the Institute of Psychiatry (King's College London), and the University of Manchester under the Directorship of Professor Til Wykes (Institute of Psychiatry) and Deputy 
J⿹

editorial
Directorship of Professor Max Marshall (University of Manchester). It represents a unique opportunity to widen participation within research and help reshape mental health practice with evidence. By fulfilling a coordinating role, we hope to raise the standard of mental health and social care research throughout England.

\section{Structure of the MHRN}

The MHRN is a managed network and its structure is shown in Figure 1. It brings together researchers and providers of mental health and social care services. The MHRN hosts, but does not fund research. Its strategy is supported by an advisory group that has representatives from Department of Health Funding agencies as well as academics and service users.

The power to run larger studies is dependent on the MHRN research hubs, which are based around England including the Midlands, Bristol, Manchester, London and Cambridgeshire. Hubs are chosen through a competitive process on the basis of academic quality, NHS partnerships, and the ability to represent different and complementary geographical and cultural constituencies to support both large randomised controlled clinical trials and projects involving rare conditions.

\section{Research creativity}

High-quality proposals in specific Department of Health priority areas will be fostered through a limited number of research groups. Each research group will have the task of developing a programme of proposals to run on the Network and to feed back issues for the Department of Health to consider in the future. They will consist of leading UK researchers, including service user researchers, who have a strong research track record in the relevant priority area. One research group in early intervention has already been established and another in self-help has just started.

\section{Involving service users}

Support for service user involvement in research will be provided by the Service User Research Group in England
(SURGE). It is responsible for encouraging collaborative efforts between service users and clinical academic and supporting both partners in large research studies.

\section{Getting research onto the MHRN}

The MHRN is an open network so it is not necessary to be a member of a hub or a research group in order to take advantage of the infrastructure. Investigators can apply to run a project on the Network by a brief submission to the Adoptions Committee. The Adoptions Committee considers the suitability of outline proposals according to the principles and criteria detailed in Box 1 .

\section{What will the MHRN add?}

We anticipate that a coordinated managed network will make large-scale mental health research easier in three key ways:

- Through research groups to create a culture of largescale studies relevant to key problems in mental health.

- Through the Hubs to provide access to a stable infrastructure of clinical /academic centres with a track record of large-scale recruitment across a large and diverse patient population.

- If successful, the Network will encourage funding bodies to view mental health research as capable of competing in quality and quantity with research in other branches of medicine.

The Medical Research Council has been responsive in this area and has already allocated new money in its Brain Sciences Initiative (trial platforms and pathfinder grants: http://www.mrc.ac.uk/index/funding.htm). We are hopeful that other funders will follow this lead and also develop partnerships to support larger, and perhaps more meaningful, studies in mental health.

\section{Conclusion}

We have no doubt that establishing the Network will be a complex and at times controversial process. The Network facilitates but does not fund research, so in the end success will depend on the desire of researchers,

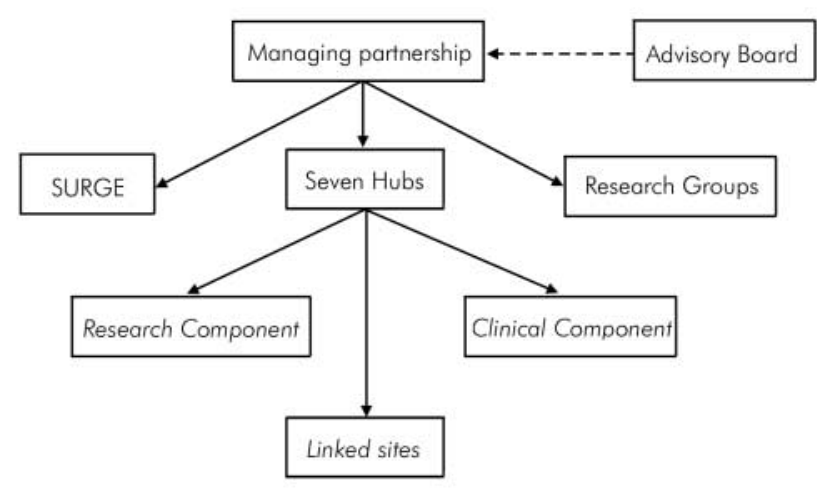

Figure 1. Structure of the Mental Health Research Network. 


\section{Box 1}

\section{Principles}

(i) there is evidence of service user input into development of the proposal;

(ii) the proposal is in line with national mental health policy;

(iii) the proposal must be free of ethical or major design flaws.

\section{Criteria}

The proposal:

(i) requires multiple centres because of a need for: a large sample size, recruitment of participants with a rare condition, or from a particular group or lifestyle, OR

(ii) is a cluster-randomised trial requiring multiple centres or units of randomisation, OR

(iii) requires multiple centres in order to establish whether a finding or an intervention is a valid generalisation across different settings or is applicable in specific settings (for example, rural areas).

clinicians and service users to work together. It is our belief that the mental health research community will be sufficiently far-sighted to make the most of this unique opportunity and realise its full potential.

\section{Declaration of interest}

The Mental Health Research Network (MHRN) is a standing programme of the National Institute of Mental Health in England (NIMHE) and is funded by the Department of Health. For more information, see www.mhrn.info

\section{References}

ACADEMY OF MEDICAL SCIENCES Primary and Secondary Care. London: (2003) Strengthening clinical research. Gaskell. London: Academy of Medical Sciences.

THORNICROFT, G. \& MAINGAY, S (2002) The global response to mental OFFICE OF SCIENCE AND TECHNOLOGY， illnesS. BMJ, 325, 608-609. DEPARTMENT OF TRADE AND INDUSTRY (2003) Bioscience 2015. Improving national health, increasing national wealth; a report to Government by the Bioscience Innovation and GrowthTeam. London: HMSO.

THORNLEY, B. \& ADAMS, C. (1998) Content and quality of 2000 controlled trials in schizophrenia over 50 years. BMJ, 317, 1181-1184.

NATIONAL INSTITUTE FOR CLINICAL EXCELLENCE (2002) Schizophrenia: Care Interventions in theTreatment and Management of Schizophrenia in TORREY, F., ZDANOWICZ, M., WOLFE, S., et al (2003) A Federal Failure in Psychiatric Research: Continued NIMH negligence in funding sufficient research on serious mental illnesses.

Center: www.psychlaws.org.

*Til Wykes Department of Psychology, Institute of Psychiatry, De Crespigny Park, PO Box 77, London SE5 8AF. E-mail: mhrn@iop.kcl.ac.uk, Max Marshall University of Manchester

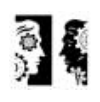

editorial 